but rather the logical conclusion of early Anabaptist thought about the nature of property and possessions.

Hutterite Beginnings also has significance for the understanding of Anabaptism which extends beyond reassigning the Hutterites and other Moravian Anabaptists to their rightful place in the history of the movement. As a melting pot of Swiss and South German Anabaptist traditions, Moravia provides the opportunity to test some basic assumptions in Anabaptist studies. Packull's analysis of the interaction of these traditions involves a revision on the polygenesis model of early Anabaptism to which he contributed significantly. This is not to suggest that polygenesis has been abandoned, but rather that it has become more nuanced to accomodate new research. Equally important are the implications of Packull's re-evaluation of the debate between Marpeck and the spiritualizing Anabaptists and its aftershocks down to the 1540 s which further erode the sharp boundaries drawn between "Spiritualists" and "Sectarians" in the Radical Reformation.

Hutterite Beginnings, like its subject matter, has significance far beyond the boundaries of the Hutterian Brüderhöfe or the borders of Moravia. It will provide stimulating and profitable reading for scholars of Anabaptism and the Reformation more generally.

\title{
GEOFFREY DIPPLE, University of Toronto
}

"Christophe Colomb et la découverte de l'Amérique. Réalités, imaginaire et réinterprétations," actes de la rencontre de la Société des Italianistes de l'Enseignement Supérieur et de la Société des Hispanistes Français, Études hispanoitaliennes, 5 (1994).

Ces actes abordent cinq aspects de la "découverte" de l'Amérique par Colomb: les influences et le milieu, l'idéologie et l'écriture, la géographie et l'imaginaire, le discours historiographique, enfin l'iconographie.

Deux "communications introductives" amorcent la réflexion. Dans la première, Ricardi García Cárcel rappelle, fort à propos dans un ouvrage publié à l'occasion du cinquième centenaire de la "découverte," les manipulations de la mémoire historique lors des précédentes commémorations. Dans la seconde, Gabriella Airaldi, qui n'a malheureusement pas tenu compte de cette mise en garde, rappelle "le parcours exemplaire" de Colomb et présente un portrait quelque peu hagiographique de l'homme.

Mais dans son analyse de "l'influence de Toscanelli et de son milieu sur Christophe Colomb," Franck La Brasca redonne au colloque sa dimension critique en proposant un ambitieux programme de recherches qui permettrait de mieux cerner "le rôle de l'humanisme philologique, philosophique et scientifique dont 
Toscanelli fut un des représentants les plus qualifiés" (p. 49). En revanche, dans "Christophe Colomb et le Portugal: État de la question," Georges Boisvert avoue n'apporter rien de neuf et se contente de reprendre à son compte les observations d'Avelino Teixeira da Mota, parues dans The Journal of the American Portuguese Cultural Society, en 1968. Ces deux communications, sans enlever à Colomb son mérite, rappellent que ses explorations n'auraient pu avoir lieu sans le brassage des idées qui avait cours à la fin du quinzième siècle et sans l'expérience maritime des Portugais.

La seconde section comprend trois études. Dans la première, Jean Lacroix analyse la rhétorique, ou plutôt l'"écriture hyperbolique" de Colomb orchestrant sa propre hiérophanie. Pour sa part, Georges Baudot s'est attaqué à un thème étudié par nombre de critiques: "le regard de Colomb sur l'Autre." À l'instar de ses devanciers, il conclut que "le regard de Colomb voit dans l'Autre ce qu'il veut d'abord, a priori, y voir, ne consent à voir que ce qu'il a prévu" (p. 89). Était-ce utile de rappeler des thèses déjà connues? Plus innovatrice, l'analyse d'Alain Milhou montre l'inflence du "messianisme" inspiré de Joachim de Fiore sur Colomb, tout en s'interrogeant sur la sincérité ou l'opportunité de l'adhésion de celui-ci à ces thèses. Mais, de rappeler Alain Milhou, il ne faut pas oublier que les schémas mentaux des hommes du quinzième et du seizième siècle différaient des nôtres.

Dans la section consacrée à la "découverte: géographie et imaginaire," Adelin Charles Fiorato s'intéresse aux "points de filiation ou de convergence" entre les utopies italiennes du seizième siècle et la découverte de l'Amérique. Selon lui, les utopies italiennes éliminent le voyage d'exploration pour ne retenir "que son point d'arrivée: l'insularité" (p. 120), et les règles d'organisation complexe et rationnelle de l'État, qui les caractérisent, se nourissent peut-être des observations de certaines civilisations amérindiennes (p. 122). Enfin, la vision eschatologique de Campanella n'est pas sans évoquer des idées de Colomb lui-même (p. 126). Les limites d'une telle analyse tiennent au fait qu'elle ne distingue pas ce qui s'inspire du modèle canonique, l'Utopie de Thomas More, voire des courants de pensées de l'époque, et ce qui provient des explorations de Colomb. Or l'invention du paradis terrestre par Colomb, analysée par Jean-Pierre Sánchez, illustre à la fois la complexité du personnage "pétri de religion et nourri d'obsessions" (p. 142), et la difficulté de distinguer ce qui est propre à Colomb de ce qui appartient aux modes de pensée de l'époque.

De loin la plus longue, la quatrième section porte sur "Colomb dans la littérature et l'historiographie," du seizième siècle au dix-huitième siècle. L'enquête de Lucien Vendrame "sur la perception qu'ont eu les Florentins des nouvelles réalités" dans la vingtaine d'auteurs de la Raccolta (p. 160) montre l'attrait qu'exercent des terres "où l'enrichissement est à portée de la main" (p. 162) en même temps que le "processus de dénigrement systématique des Indiens" fondé sur le 
sentiment de supériorité des Européens (p. 163). L'étude de L. Vendrame est d'autant plus intéressante qu'elle témoigne des premières réactions des Européens au lendemain de la découverte de l'Amérique, alors que se constitue une vision négative de l'Amérindien, trop souvent occultée par l'importance accordée aux Essais de Montaigne.

Dans son analyse des strophes du Roland furieux consacrées à la découverte de l'Amérique, au voyage de Roger au-delà des Colonnes d'Hercule et à celui du poète dans son imaginaire, Roger Baillet fait des rapports harmoniques entre l'esprit de découverte et l'entreprise poétique une caractéristique de la Renaissance.

Tout comme la poésie, l'histoire n'échappe pas à l'influence de la "découverte." En témoigne Guichardin qui, dans la Storia d'Italia, "n'opère pas un repli de la politique vers l'histoire conçues comme activités de lettré, mais une poursuite de son action politique par l'écriture" (p. 179). À la vérité, ce que montre surtout Perle Abbrugiati, ce sont les motivations politiques de Guichardin, qui veut notamment "fournir une explication à la politique de Terre ferme que Venise" entend accentuer (p. 186), et ses motivations symboliques, lorsqu'il "se sert de l'épisode colombien pour renforcer l'idée de crise comme idée maîtresse de l'histoire" (p. 186).

"L'image de Christophe Colomb dans les De Orbe Nova Decades de Pierre Martyr d'Anghiera" permet à Paul Roche de montrer à quel point l'écrivain italien a adopté un ton cavalier, sinon méprisant à l'endroit de Colomb, en plus d'omettre nombre de "détails" (p. 193), voire de les présenter de manière "ambiguë" (p. 194). Si cette analyse, amorcée naguère par Paul Gaffarel, demeure juste, le lecteur reste sceptique lorsque Paul Roche explique que Pierre Martyr d'Anghiera, "trop proche du pouvoir pour prendre le risque de lui déplaire, s'est efforcé - et il y est parvenu - à traiter par l'indifférence un personnage mal en cour que sans doute au fond de lui-même il admirait" (p. 198). Cette thèse, pour séduisante qu'elle soit, n'est malheureusement pas étayée.

Dans son étude du "statut de l'épisode colombien dans la Historia general de las Indias de Francisco López de Gómara," Monique Mustapha montre comment l'aventure colombienne a servi de "canevas" à l'historien espagnol pour bâtir les épisodes ultérieurs de son ouvrage. De là une "vision" de l'histoire fondée sur l'idée que "la conquête est un perpétuel recommencement" (p. 202), ce qui n'exclut pas des visées politiques chez Gómara, qui "rabaisse Colomb pour mieux exalter les Espagnols" (p. 207). Toutefois, les différentes manifestations du "modèle" ne sont guère analysées et les rapports entre ceux-ci et les visées politiques de l'historien ne sont pas vraiment explicitées.

Dans son analyse de la "récurrence d'un schéma dantesque," Brigitte Urbani rappelle qu'au seizième siècle, l'Iliade, l'Énéide et la Jérusalem délivrée servirent de modèles aux écrivains qui évoquaient la figure de Colomb. Mais après qu'Alessandro Tassoni eut dénoncé les incohérences issues de ces modèles et suggéré de 
s'inspirer plutôt de l'Odyssée, les poètes ont emprunté à la fois au texte d'Homère et à l'épisode du dernier voyage d'Ulysse, dans la Divine Comédie, pour rendre compte de l'expérience de Colomb.

L'étude des "images de Christophe Colomb en Italie, des Lumières à Leopardi" permet à Bruno Toppan de rappeler que c'est précisément sur l'ingratitude et sur l'incompréhension, qui ont accompagné l'entreprise de Colomb, que se fondent les symboles et le sens philosophique des discours tenus sur celui-ci, soit dans une perspective utilitariste (Giuseppe Parini), soit dans une perspective qui promeut la rationalité (Alessandro Verri). Mais ces auteurs ne perdent pas tout sens critique et ils ne manquent pas de dénoncer les conséquences négatives de la découverte de l'Amérique, thème qu'on retrouve, bien sûr, chez Leopardi, qui fait de Colomb un homme ébranlé par le doute.

Dans "Christophe Colomb, l'Amérique et la littérature française du XVIII siècle," Jean-Pierre Clément rappelle que la littérature de voyage répond à un besoin d'évasion et d'exotisme, qui n'exclut pas la réflexion philosophique et politique. La communication n'innove en rien sur ce qu'a écrit naguère Gilbert Chinard, alors qu'il eût été intéressant d'analyser les variations qu'a connues la figure du "Sauvage" au cours du siècle. Entre Adario, promu au rang de "philosophe" par Lahontan et l'homme primitif, conçu par Jean-Jacques Rousseau, le discours sur l'Autre a sensiblement évolué. Il aurait été intéressant aussi d'étudier comment les "philosophes" ont su confisquer aux missionnaires la figure de l' Américain" forgée dans les Relations du dix-septième siècle. Enfin, dans sa communication, Charles Minguet analyse le travail d'Alexander Von Humboldt sur Vespucci et sur Colomb pour montrer à quel point ses observations demeurent justes, notamment à cause de la méthode pour étudier l'histoire qu'elles appellent.

La dernière section, "Colomb dans l'iconographie," ne comprend qu'une étude, celle de Jean-Paul Duviols, qui porte sur 28 représentations du débarquement de Colomb à Guanahani et qui rend compte de l'"élaboration progressive d'un stéréotype" (p. 262). J.-P. Duviols analyse avec finesse les documents qu'il produit et montre comment certains graveurs recréent l'événement en empruntant des détails à des gravures qui n'ont rien à voir avec l'Amérique. Seul regret: l'auteur n'aborde pas la question de la physionomie des Amérindiens, désespérément européenne jusqu'à la fin du dix-neuvième siècle. Il est vrai que le temps et l'espace étaient chichement mesurés et que Jean-Paul Duviols a dû s'en tenir à l'essentiel, ce qu'il a fort bien fait.

En guise de conclusion, rappelons, à la suite de Pier Luigi Crovetto, que la découverte du Nouveau-Monde s'est déroulée sur fond de controverse, de rêve et d'intérêts bien concrets. En témoignent les communications publiées dans ce numéro des Études hispano-italiennes.

PIERRE BERTHIAUME, Université Laval 